\title{
The key regulator LCERF056 enhances salt tolerance by modulating reactive oxygen species-related genes in Lotus corniculatus
}

\author{
Dan Wang ${ }^{1,2+}$, Zhanmin Sun ${ }^{1 *}{ }^{*}$, Xinxu Hu ${ }^{3,7 \dagger}$, Junbo Xiong ${ }^{4}$, Lizhen $\mathrm{Hu}^{5}$, Yuandong $\mathrm{Xu}^{6}$, Yixiong Tang ${ }^{1 *}$ and \\ Yanmin $\mathrm{Wu}^{1 *}$
}

\begin{abstract}
Background: The APETALA2/ethylene response factor (AP2/ERF) family are important regulatory factors involved in plants' response to environmental stimuli. However, their roles in salt tolerance in Lotus corniculatus remain unclear.

Results: Here, the key salt-responsive transcription factor LCERF056 was cloned and characterised. LCERF056 belonging to the B3-1 (IX) subfamily of ERFs was considerably upregulated by salt treatment. LcERF056-fused GFP was exclusively localised to nuclei. Furthermore, LCERF056- overexpression (OE) transgenic Arabidopsis and L. corniculatus lines exhibited significantly high tolerance to salt treatment compared with wild-type (WT) or RNA interference expression (RNAi) transgenic lines at the phenotypic and physiological levels. Transcriptome analysis of OE, RNAi, and WT lines showed that LCERF056 regulated the downstream genes involved in several metabolic pathways. Chromatin immunoprecipitation-quantitative polymerase chain reaction (ChIP-qPCR) and yeast one-hybrid (Y1H) assay demonstrated that LCERF056 could bind to cis-element GCC box or DRE of reactive oxygen species (ROS)-related genes such as lipid-transfer protein, peroxidase and ribosomal protein.
\end{abstract}

Conclusion: Our results suggested that the key regulator LCERF056 plays important roles in salt tolerance in $L$. corniculatus by modulating ROS-related genes. Therefore, it may be a useful target for engineering salt-tolerant $L$. corniculatus or other crops.

Keywords: ERF transcription factor, Salt stress, Regulation mechanism, Lotus corniculatus

\section{Background}

Salinity is one of the most widespread abiotic stresses that limits plant growth and crop productivity. Approximately $7 \%$ of the land area and $19.5 \%$ of arable lands worldwide are under salt stress [1]. Salt exerts detrimental effects on plants by causing osmotic stress, ion imbalance, and oxidative toxicity. To survive salt stress, plants

\footnotetext{
*Correspondence: sunzhanmin@caas.cn; tangyixiong@caas.cn; wuyanmin@caas.cn

${ }^{\dagger}$ Dan Wang, Zhanmin Sun and Xinxu Hu contributed equally to this work.

${ }^{1}$ Biotechnology Research Institute, Chinese Academy of Agricultural Sciences, Beijing, China

Full list of author information is available at the end of the article
}

have evolved to have high plasticity and elaborate mechanisms, comprising osmotic stress resistance, ion exclusion, and tissue tolerance. The stress-related molecular regulatory network is complex and mostly unexplored [2]. Hence, exploring the key and novel genes that regulate salt stress-related genes has become increasingly important in modern agriculture.

As key regulators, transcription factors (TFs) have crucial functions in plant growth and development and environmental stress responses. The APETALA2/ ethylene responsive factor (AP2/ERF) is one of the most important families of plant-specific TFs. It comprises a well-conserved AP2/ERF domain consisting of 
50-70 amino acid residues, and it binds to some specific cis-acting elements such as dehydration-responsive element (DRE) and GCC box for the regulation of expression of many downstream genes [3].

In recent years, many AP2/ERF genes involved in inducing abiotic or biotic stress response have been reported. For instance, IbRAP2-12, from the salttolerant sweet potato, was induced by $\mathrm{NaCl}$, PEG, $\mathrm{ABA}$, and MeJA. IbRAP2-12-overexpressing Arabidopsis lines were found to be more tolerant to salt and drought stresses than wild-type (WT) plants [4]. Overexpression of GsERF7 and GsSnRK1 synergistically increased the tolerance of transgenic Arabidopsis plants to salt-alkali stress [5]. Overexpression of ERF1-V in wheat could improve resistance to powdery mildew, salt, and drought stress [6]. In addition, AP2/ERF TFs have been found to have close association with plant growth and development and hormone responses. OsERF48 was reported to enhance root growth and drought tolerance by regulating a calmodulin-like protein gene OsCML16 [7]. Arabidopsis overexpressing AtERF019 was reported to have a 7-day delay in flowering, a 2-week delay in senescence, and increased tolerance to water deprivation when compared with WT plants [8]. Rice ethyleneresponse AP2/ERF factor OsEATB can restrict internode elongation by downregulating a gibberellin biosynthetic gene [9]. These results indicated that the AP2/ERF genes exhibit various functions and play crucial roles in the growth, development, and biotic or abiotic stress responses in plants.

L. corniculatus (Birds foot trefoil), a member of perennial legume forage, is considered to be one of the most agriculturally important forage plants due to their major agricultural advantages, including the anti-bloating properties because of tannin content and ability to grow in low fertile, acidic, and highly saline soils, and it is widely used to prevent roadside erosion. Previously, we isolated and identified 127 AP2/ERF genes from $L$. corniculatus. Expression profile analysis of these AP2/ERF genes by quantitative real-time PCR (qPCR) revealed that 19 LcERF genes were significantly upregulated by salt stress. Among these genes, LcERF054, LcERF080, and LcAP2/ERF107 were found to significantly enhance the tolerance to salt stress in transgenic Arabidopsis plants [10-12]; however, the molecular mechanism remains unclear.

To understand the molecular mechanisms underlying salt tolerance in L. corniculatus in detail, we conducted the characterisation and function analysis of LCERF056 and further assessed the mechanisms of action and regulatory pathways that affect salt stress response in L. corniculatus.

\section{Results}

\section{Molecular characteristics of LCERF056}

The full-length cDNA of LcERF056 (GenBank accession KC777345) was isolated from L. corniculatus. The gene contained a 558-bp open-reading frame and encoded a protein with 185 amino acid residues. Additionally, genomic DNA containing the $5^{\prime}$ and $3^{\prime}$ untranslated regions of LcERF056 was sequenced. No introns were found in the genomic LcERF056 upon comparison with the cDNA and genomic DNA sequences (data not shown). Analysis of the amino acid sequence of LcERF056 showed that the protein contained a single AP2/ERF DNA-binding domain (amino acids 121-179) (Fig. S1A). Phylogenetic tree (Fig. S1B) analysis suggested that LcERF056 was closely related to AtERF013 (with a $35.55 \%$ sequence identity) and belonged to the B3-1 (IX) group of ERF transcription factor family (Fig. S1C).

\section{Expression pattern of LCERF056}

To investigate the spatial expression patterns of LCERF056, RT-PCR was performed with mRNAs extracted from different organs, including roots, stems, leaves, flowers, and seeds of L. corniculatus. LcERF056 was found to express in the stems and leaves, whereas its expression was not detected in the roots, flowers, or seeds (Fig. 1A). To study the effects of different stress signals on gene expression, $L$. corniculatus seedlings were treated with ABA, ACC, JA, SA, PEG, or NaCl. RNA extracted from the seedlings at 0,3 , and $24 \mathrm{~h}$ after the treatments were subjected to qPCR to evaluate the mRNA levels of LCERF056 upon exposure to various stress signals. The results (Fig. 1B) showed that mRNA of LcERF056 could be detected in all cases after 3 and $24 \mathrm{~h}$. In seedlings treated with $\mathrm{NaCl}(24 \mathrm{~h})$, LcERFO56 was significantly upregulated (110-fold higher than the WT). Additionally, JA treatment could increase the mRNA levels by 24-fold at $3 \mathrm{~h}$, whereas $\mathrm{ABA}$ and SA treatments exhibited only a slight effect on the mRNA levels of LcERF056. After ACC and PEG treatments, LcERF056 mRNA levels were found to decrease significantly at 3 and $24 \mathrm{~h}$.

\section{LcERF056 localises to the nucleus and acts as a transcriptional activator}

To examine the subcellular localisation of LcERF056, LCERF056 was fused to the green fluorescent protein (GFP) gene driven by CaMV35S promoter. The fused gene 35S:LcERF056-GFP was transiently expressed in Arabidopsis protoplast. As a control, similar experiment without LcERF056 fusion (GFP gene only) was conducted at the same time. LcERF056-GFP was found to be localised in the nucleus, whereas the control GFP was distributed throughout the cells (Fig. 2A). Further, to 

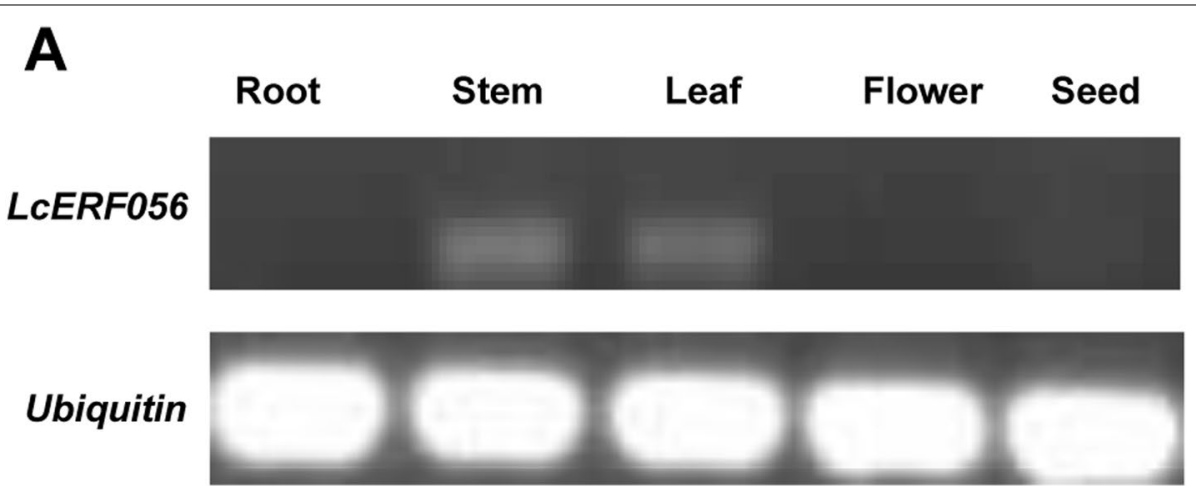

B

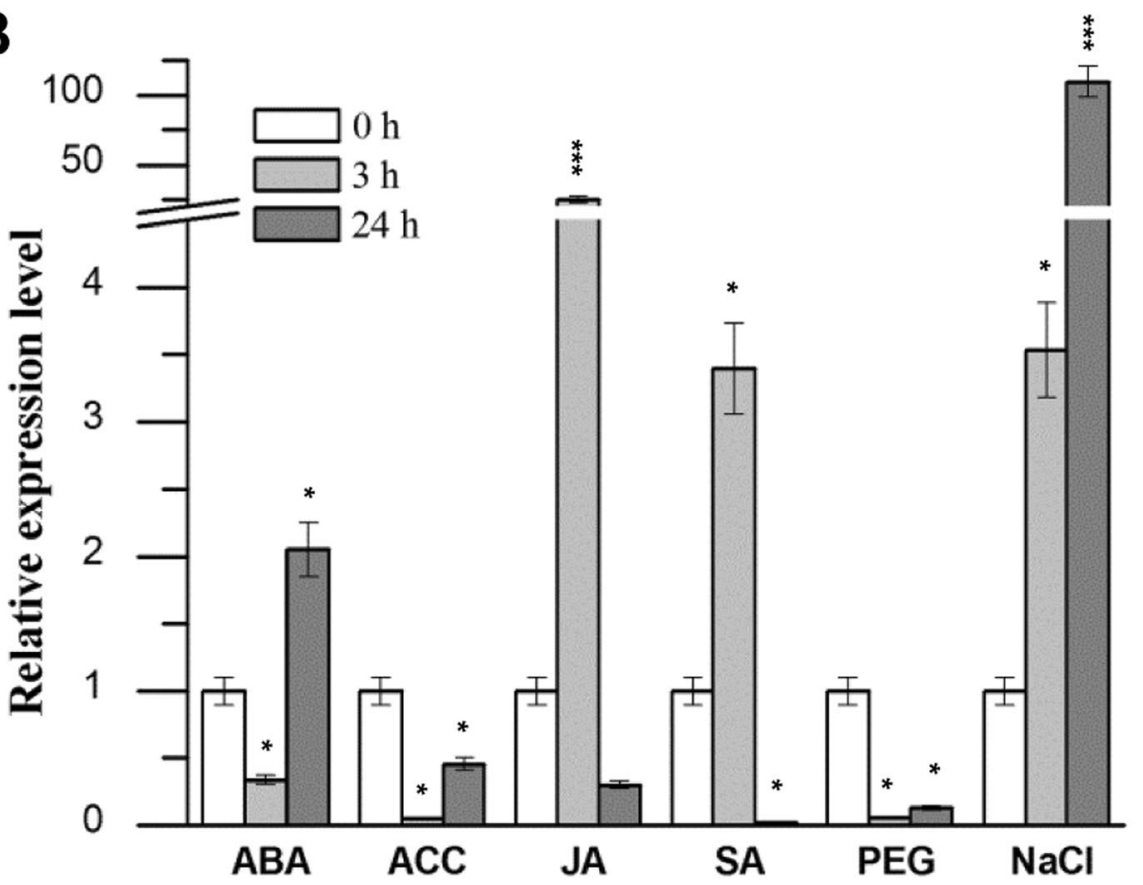

Fig. 1 The expression patterns of LCERF056 mRNA. (A) Expression of LCERF056 mRNA in various tissues evaluated using RT-PCR. (B) Expression level of LCERF056 in response to abiotic stresses. Four-week-old seedlings were exposed to $100 \mu \mathrm{M}$ of ABA, ACC, JA, SA, 20\% PEG, or $150 \mathrm{mM} \mathrm{NaCl}$. The relative expression was calculated using polyubiquitin as an internal reference. The WT unstressed expression level was assigned a value of 1. Data represent the averages of three independent experiments \pm SE

understand the function of LcERF056, yeast one-hybrid $(\mathrm{Y} 1 \mathrm{H})$ assay was performed using the construct carrying LcERF056. Results of the $\mathrm{Y} 1 \mathrm{H}$ assay demonstrated that LcERF056 could act as an activator for the transcription of genes (Fig. 2B).

\section{Overexpression of LCERF056 in Arabidopsis enhances salt tolerance}

No obvious morphological difference was observed between the WT plants and all the 10 lines of 35S:LcERF056 transgenic Arabidopsis plants. However, the 4-week-old 35S:LcERF056 plants exhibited higher tolerance to salt stress than the WT after they were treated with $\mathrm{NaCl}$ for 21 days (Fig. 3A). The relative moisture content was lower in WT than in the transgenic plants (Fig. 3B). The relative membrane ion leakage, an index for the level of cellular damage, was significantly lower in 35S:LcERF056 plants than in WT plants (Fig. 3C). The results suggested that $L c E R F 056$ was involved in defence of salt stress, and ectopic expression of LcERF056 could increase the salt tolerance in Arabidopsis.

\section{LCERF056 enhances defence against salt stress in $L$.} corniculatus

To explore the endogenous function of LcERF056 in L. corniculatus, LcERF056 knockdown experiments were conducted. For comparison, LcERF056 was overexpressed in L. corniculatus at the same time. RNA 


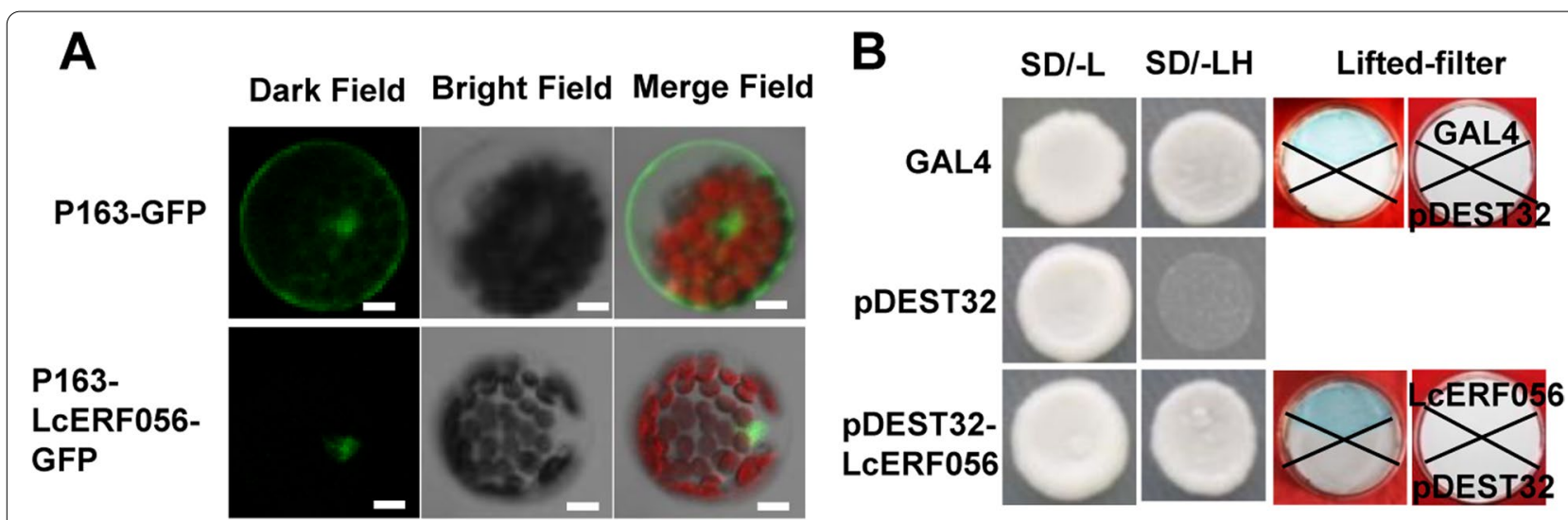

Fig. 2 LCERF056 is a nuclear protein acting as a transcriptional activator. (A) Subcellular localisation of LCERF056. Plasmids containing 35S:GFP and 35S:LcERF056-GFP were introduced into Arabidopsis protoplast by polyethylene glycol transfection. Nuclear localisation was investigated through confocal microscopy. Bars $=5 \mu \mathrm{m}$. (B) Analysis of transcriptional activation of LcERF056 by the yeast one-hybrid assay

A

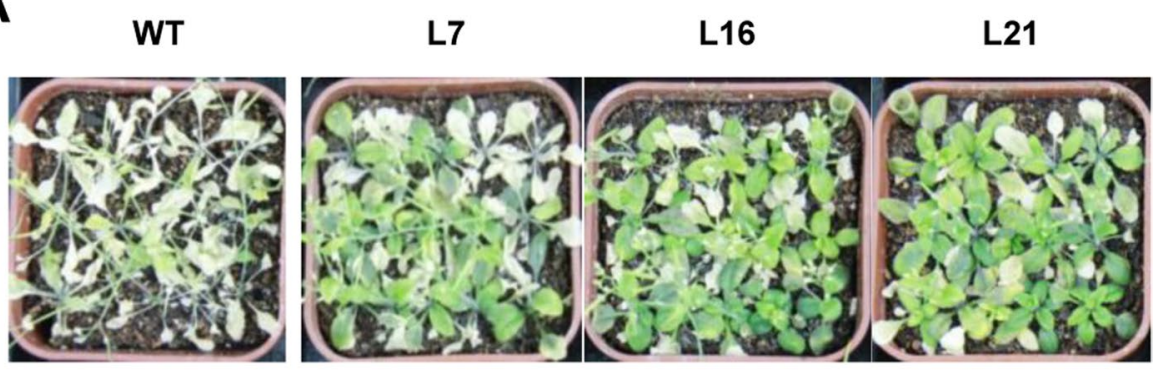

$300 \mathrm{mM} \mathrm{NaCl}$ stress for $21 \mathrm{~d}$

B

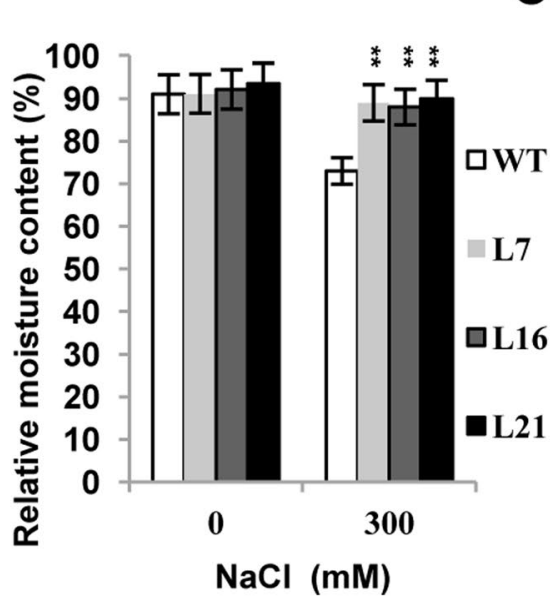

C

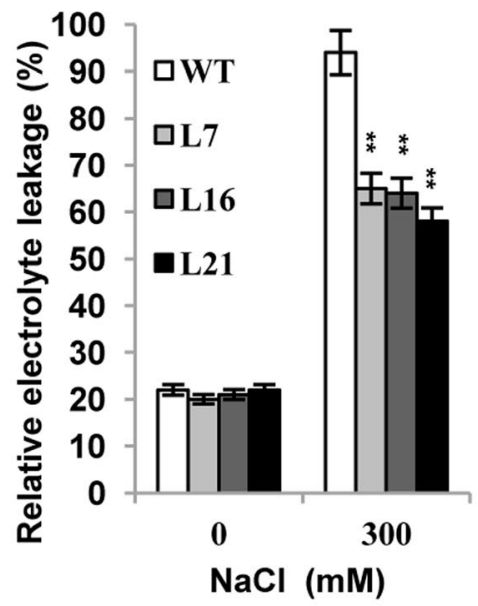

Fig. 3 Overexpression of LCERF056 confers enhanced salt tolerance in transgenic Arabidopsis. (A) Growth of WT and transgenic plants under $300 \mathrm{mM} \mathrm{NaCl}$ stress after 21 days. Ten-day-old seedlings grown on MS medium plates were planted in pots filled with a 1:1 mixture of vermiculite and humus and well-watered for 20 days. The seedlings were watered with a solution containing $300 \mathrm{mM} \mathrm{NaCl}$ for 21 days. The experiment was repeated three times. (B) Relative moisture contents. (C) Relative electrolyte leakage. The data represent the means of three replicates. The error bars indicate the SD. ${ }^{* *} P<0.01$ compared with the WT plants, using Student's t test 
interference plants (LcERF056-RNAi) and overexpressing plants (LCERFO56-OE) of L. corniculatus were obtained through Agrobacterium-mediated transformation. The presence of the transgenes in the putative transgenic $L$. corniculatus was examined through PCR genotyping. As shown in Fig. S2, the transcript of LcERF056 greatly decreased in the LcERF056-RNAi lines and increased significantly in the LcERFO56-OE lines compared with that in WT plants. The phenotypes were characterised using in vitro tissue culture plants (Fig. S3) because no seeds could be obtained when LCERFO56-OE plants were grown in the laboratory or greenhouse. The LcERF056OE lines exhibited a significantly lower plant height but more branches than the WT at 90 days, whereas a significantly higher plant height and fewer branch numbers were observed in LcERF056-RNAi lines (Fig. S3).

To examine whether LcERF056 is involved in defence against salt stress in $L$. corniculatus, we treated WT, LcERF056-RNAi, and LcERF056-OE plants, which had been transplanted into pots for 10 days in advance, with $250 \mathrm{mM} \mathrm{NaCl}$ solution. Salt stress considerably inhibited the plant growth (Fig. 4A). After 15 days, approximately half of the leaves of LcERF056-RNAi plants turned yellow, whereas the leaves of WT and LcERF056-OE lines remained green. The LcERF056-RNAi plants died after 25 days; however, only some of the leaves of the WT plants turned yellow and those of the LCERFO56- OE plants remained green (Fig. 4A). Further analysis showed

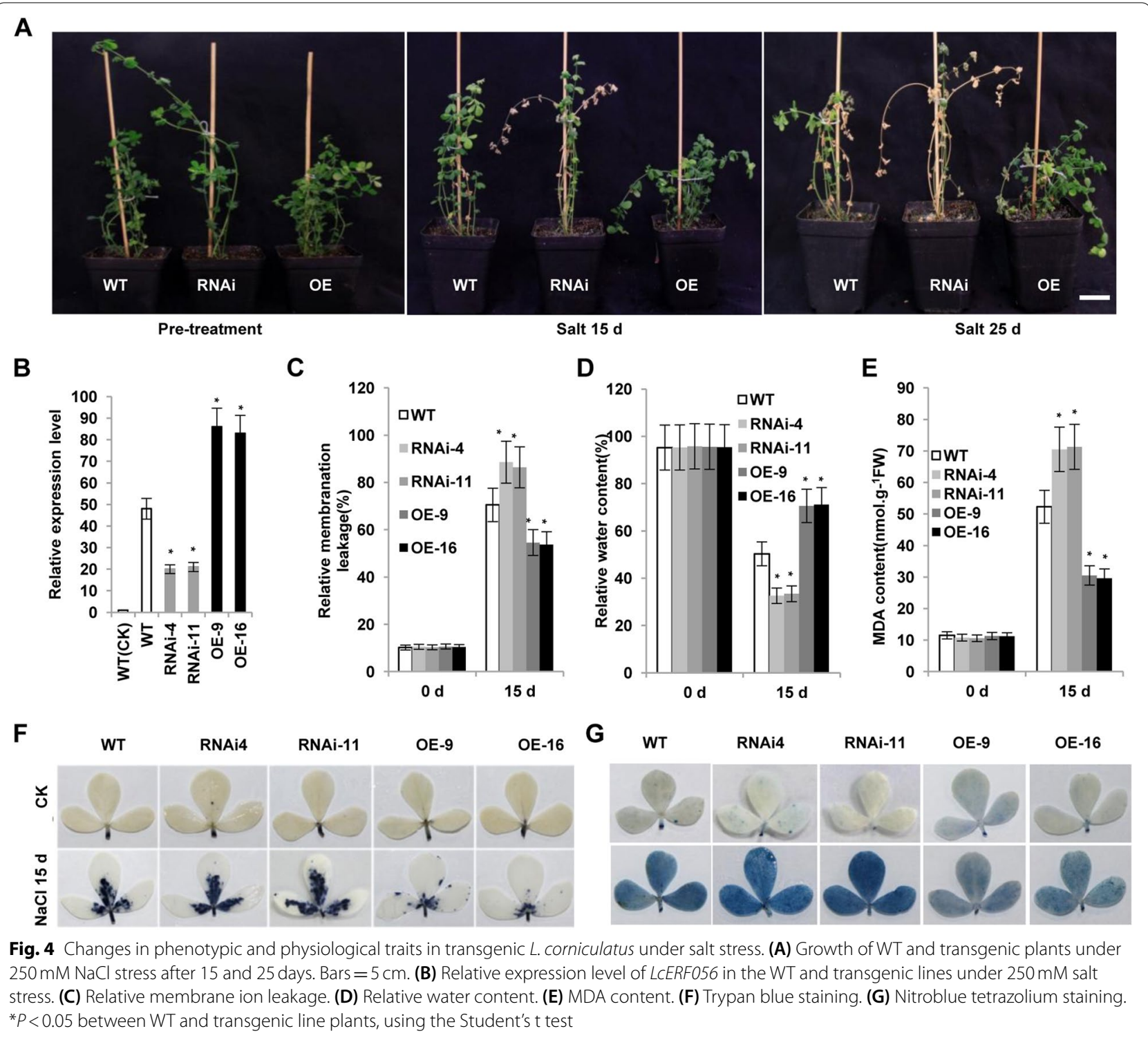



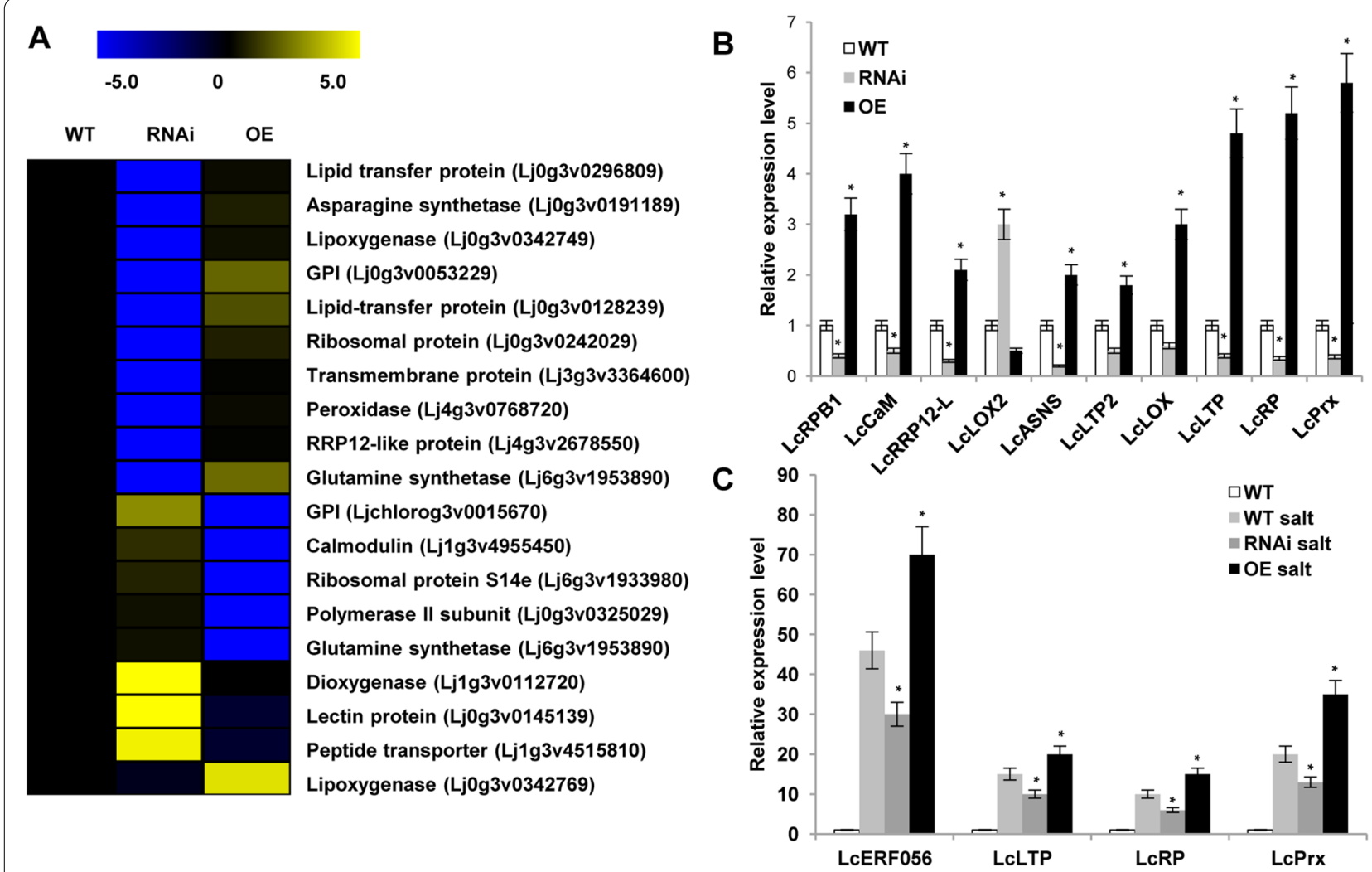

Fig. 5 Differentially expressed genes in WT, LCERF056-OE, and LCERF056-RNAi transcriptome. (A) Heatmap of 19 genes related to growth and stress. (B) Expression patterns of 10 genes related to growth and stress in WT, LCERF056-OE, and LCERF056-RNAi lines. (C) The relative expression level of the 3 target genes in WT, LCERF056-OE, and LCERF056-RNAi lines under salt treatment. The WT expression level was assigned a value of 1. Data represent the averages of three independent experiments $\pm \mathrm{SE}$

that the membrane ion leakage increased significantly in the LCERF056-RNAi lines and decreased in the LCERFO56-OE lines in comparison with the WT plants (Fig. 4C). Other salt-stress-tolerance indices, such as lipid peroxidation levels represented by malondialdehyde (MDA) content, relative water content, accumulation of superoxide ion superoxide $\left(\mathrm{O}_{2}^{-}\right)$, and cell death inferred by trypan blue staining (Fig. 4D-G), were assessed. Under salt stress, LcERF056-RNAi plants showed significantly lower water content, higher MDA content, higher accumulation of $\mathrm{O}_{2}^{-}$, and enhanced cell death. Conversely, LCERFO56-OE plants exhibited significantly higher water content, lower MDA content, lower accumulation of $\mathrm{O}_{2}^{-}$, and lower cell death (Fig. 4D-G). The results suggested that LcERF056 played a crucial role in salt stress defence in L. corniculatus.

\section{LCERF056 overexpression leads to extensive transcription of stress-responsive genes}

To investigate the underlying molecular mechanism of salt stress defence mediated by LcERF056, RNA-Seq of the WT, LcERF056-RNAi, and LcERF056-OE plants was performed. Differential expression analysis identified 47 upregulated and 80 downregulated genes $(\mathrm{P}<0.05)$ in LCERF056- RNAi plants compared with the WT plants. In LCERFO56-OE plants, 44 upregulated and 76 downregulated genes were detected (Fig. S4A). Results of the gene ontology (GO) analysis suggested that these genes could regulate multiple biological processes, including regulation of transcription, plant response to stresses, and growth (Fig. S4B, C).

To verify the candidate target genes, expression levels of the overlapped 18 genes (Table S3, S4) were analysed. Ten out of the 18 genes were further selected for qPCR analysis using L. corniculatus ubiquitin as the reference gene. The measured expression levels of most genes were highly consistent with the levels determined by RNA-Seq (Fig. 5A, B).

\section{Identification and validation of LcERF056-regulating} target genes by direct binding to motifs by using yeast one-hybrid (YIH) assay and ChIP-qPCR

Promoters of the aforementioned 10 DEGs were isolated and analysed by PlantCARE. Promoters of three genes, LcLTP, (lipid-transfer protein, LTP, Lj0g3v0128239), 
LcRP (ribosomal protein, RP, Lj0g3v0242029), and LcPrx (peroxidase, Lj4g3v0768720), were found to have the DRE element or GCC box (Table S6). Expressions of LcLTP, LcPrx, and LcRP in LcERF056-OE and LcERF056RNAi plants under salt stress were analysed using qPCR. For comparison, the same experiment was conducted using the WT line as control. The results indicated that $L c L T P, L c R P$, and $L c P r x$ were significantly downregulated in LCERF056-RNAi plants and highly upregulated in LCERF056-OE plants, consistent with the expression patterns of the LcERF056 gene (Fig. 5C). These three genes are known to be closely related to salt stress. Lipid-transfer proteins (LTPs), a class of small, ubiquitous proteins, play critical roles in various environmental stresses [13]. $R P$ genes have been shown to be differentially regulated by abiotic and biotic environmental factors $[14,15]$. Peroxidase plays prominent roles in antioxidant responses and stress tolerance in plants [16].

To study whether LcERF056 could directly bind to the promoters of these genes, $\mathrm{Y} 1 \mathrm{H}$ assay of LcERF056 was performed. The results showed that yeast cells harbouring LcERF056 and GCC box grew well in the presence of aureobasidin A (AbA), whereas cells with mutated GCC box could not survive (Fig. 6A, B). To further assess the interactions between LcERF056 and the GCC box in vivo, LcERF056 antibody was produced in advance in the present study. Proteins were extracted from L. corniculatus and subjected to western blotting. Only a single band around $22.2 \mathrm{kDa}$ was observed for each sample (Fig. S5), demonstrating the specificity of the LcERF056 antibody. The chromatin immunoprecipitation (ChIP) qPCR revealed that the LcLTP, LcRP, and LcPrx promoter enrichment fold were significantly higher in the LCERF056-OE plants than in the WT or LcERF056-RNAi plants (Fig. 6C, D). These observations suggested that the binding of LcERF056 on the promoters of LcLTP, LcPrx, and $L c R P$ was positively correlated with its transcript levels.

\section{Discussion}

LCERF056 is the key salt-responsive TF in L. corniculatus

AP2/ERF is one of the most important families of plantspecific TFs responsible for plant growth, development, and abiotic or biotic stress response $[17,18]$. This superfamily consists of several members, and to date, over 14 billion members have been identified in Arabidopsis, rice, soybean, and L. corniculatus $[10,19,20]$. However, the members playing a critical role in response to salt stress have not been identified yet. Thus, identifying the essential salt-responsive genes and investigating the underlying mechanisms are essential.
Overall, $127 A P 2 / E R F$ genes have been identified from $L$. japonicus genome [10]. To investigate if these L. corniculatus AP2/ERF genes are involved in defence against salt stress, we analysed the expression of 106 $A P 2 / E R F$ genes in response to salt treatment. Nineteen of these $A P 2 / E R F$ genes exhibited differential expression (fold change $>2, P<0.05$ ). Among them, LcERFO78 and LcERF056 exhibited 300- and 110-fold higher expression, respectively, which indicated that these two genes are potentially involved in defence against salt stress. The 19 $A P 2 / E R F$ genes were cloned into a binary vector driven by CaMV35S promoter and transferred into Arabidopsis, and independent transgenic lines for the 19 genes were obtained. Because the seeds yielded by the $\mathrm{T} 1$ generation of transgenic line expressing LCERFO78 could not yield normal seeds, no further experiments were performed on LcERFO78 in this study. Transgenic Arabidopsis plants overexpressing the other 18 genes could yield seeds normally, and no obvious morphological differences were observed in the aerial tissues as compared with the WT plants.

After treatment with $\mathrm{NaCl}$ for 21 days, the 4-week-old LCERF056-OE Arabidopsis plants exhibited marked tolerance to salt stress as compared with the WT (Fig. 3). Results of further experiments with LcERF056-OE and LCERF056-RNAi L. corniculatus plants revealed that the expression of LcERF056 could enhance salt tolerance in L. corniculatus, thus suggesting that LcERF056 is one of the key salt-responsive TFs in L. corniculatus.

\section{LCERF056 probably affects plant development}

In addition to the observation that LcERF056 was involved in response to salt stress, expression of LcEFR056 was negatively correlated with the plant height and positively correlated with branching number in L. corniculatus (Fig. S3). LcERF056-OE transgenic $L$. corniculatus lines exhibited dwarf phenotypes, indicating that the internodal elongation process was suppressed by LCERF056 overexpression. Similar phenomenon appeared in rice AP2/ERF factor OsEATB. Qi has reported that OsEATB could restrict internode elongation by downregulating a gibberellin biosynthetic gene [9], whereas, Agarwal et al. suggested that the AtERF11 TF promoted internode elongation by activation of gibberellin biosynthesis and signalling [21]. Bajaj identified that ERFs were one of the candidate genes for dissecting complex branch number trait in chickpea [22]. Plant shoot branching and height are important traits that affect forage grass production. However, there is no evidence that LcERF056 directly regulates the downstream factors of GA biosynthetic gene. Further studies are 

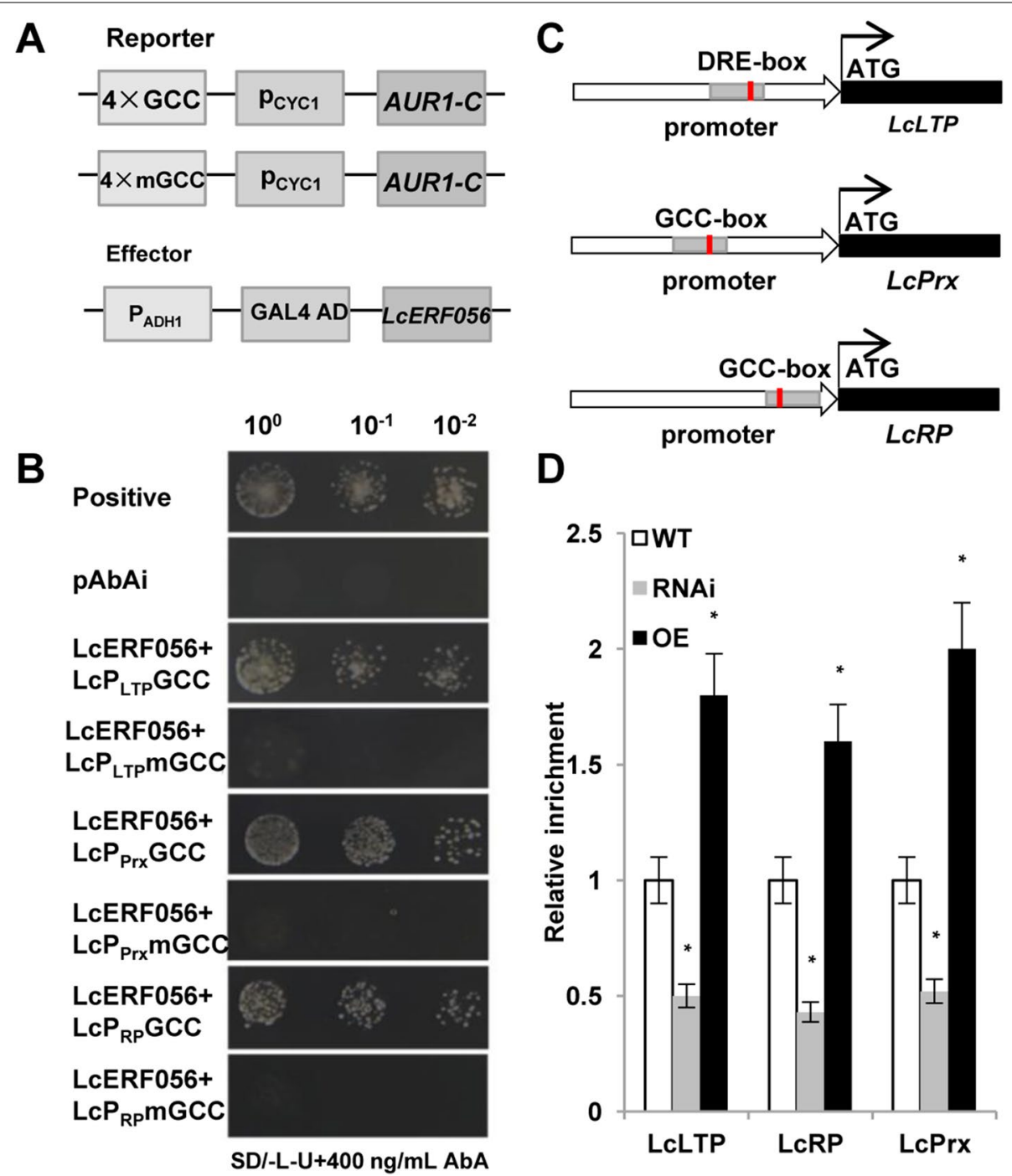

Fig. 6 LCERF056 binds directly to GCC-box and DRE motifs in promoters to activate the transcription of downstream target genes. (A) Reporter and effector vectors used for yeast one-hybrid $(\mathrm{YIH})$ experiments. (B) Verification of the interactions between LCERF056 and GCC-box and DRE cis elements through $\mathrm{YIH}$ experiments. Positive transformants were identified by spotting serial dilutions of yeast (1:1, 1:10, and 1:100) onto SD/-L-U plates supplemented with $400 \mathrm{ng} / \mathrm{mL}$ AbA. (C) Schematic structure of LCLTP, LCRP, and LCPrx genes. ATG, translation start point; Red line, GCC-box or DRE-box; Grey boxes, ChIP-qPCR amplified fragments that correspond to the regions indicated in the gene structure. (D) ChIP-qPCR analysis of the enrichment levels of LCLTP, LCRP, and LCPrx in LCERF056-OE and LCERF056-RNAi transgenic plants by using the specific synthetic antibody (rabbit). WT assay was included as control. ${ }^{*} P<0.05$ between WT and transgenic plants, using Student's t test

required to assess the roles of LcERF056 in internodal elongation and branch number.

\section{LCERF056 enhanced salt tolerance by binding} to the cis-element of ROS-related target genes

ERF subfamily genes play various roles in response to various environmental stress factors [8, 23, 24]. Previous studies have established that the AP2/ERF family regulates the transcriptional expression of downstream genes by binding to GCC box or DRE element [25, 26]. Our RNA-Seq data revealed that LcERF056 regulated the expression of many genes related to biotic stress. Additionally, we analysed the cis-element in the promoters of the candidate genes and verified that LcERF056 could bind directly to promoters of $L c L T P, L c R P$, and $L c P r x$. Using tblastn programme at website (https://blast.ncbi.nlm.nih.gov/Blast.cgi), we 
found that LcLTP displayed a high homology with nonspecific lipid-transfer protein 1 in Medicago truncatula (XP_003623613.3), soybean (XP_028213126.1), and Cicer arietinum (XP_004492552.1).

Plant LTPs are assumed to play important roles in membrane biogenesis, phospholipid transport, and biotic and abiotic stress response [27]. High LTP expression was associated with increased plant tolerance to cold, drought, and elevated hydrogen peroxide $\left(\mathrm{H}_{2} \mathrm{O}_{2}\right)$ levels [28-30]. Peroxidases play prominent roles in antioxidant responses and stress tolerance in plants by catalysing $\mathrm{H}_{2} \mathrm{O}_{2}$ oxidoreduction, wherein they facilitate transfer of electrons from various donor molecules [16]. PtrERF109 in Poncirus trifoliate was reported to contribute to cold tolerance by directly regulating the expression of Prx1, which is involved in antioxidative processes [31]. LjGpxs 1 and LjGpxs3 perform major antioxidative functions in nodules, preventing lipid peroxidation and other oxidative processes at different subcellular sites of vascular and infected cells [32]. Collectively, LTP and Prx are considered to be important stress-responsive markers in scavenging ROS.

$R P$ genes have been shown to be differentially regulated by environmental factors, both abiotic and biotic, which directly affect the plant growth and transcriptional regulation of $R P$ genes and ultimately ribosome biogenesis. Transgenic rice overexpressing RPL23A conferred water usage and tolerance efficiency to drought and salt stresses [15]. Plant ribosomal proteins, RPL12 and RPL19, were reported to play a role in nonhost disease resistance against bacterial pathogens in Nicotiana benthamiana and Arabidopsis [33]. These reports indicated that $L c E R F 056$ can enhance salt stress by regulating the $R P$ gene.

Based on the results, a functional model of LcERF056 response to salt stress was proposed (Fig. 7). LcERF056 may have a unique value in its ability to confer salt tolerance to $L$. corniculatus and other plant species because of its considerable upregulation in response to salt. The key salt-responsive

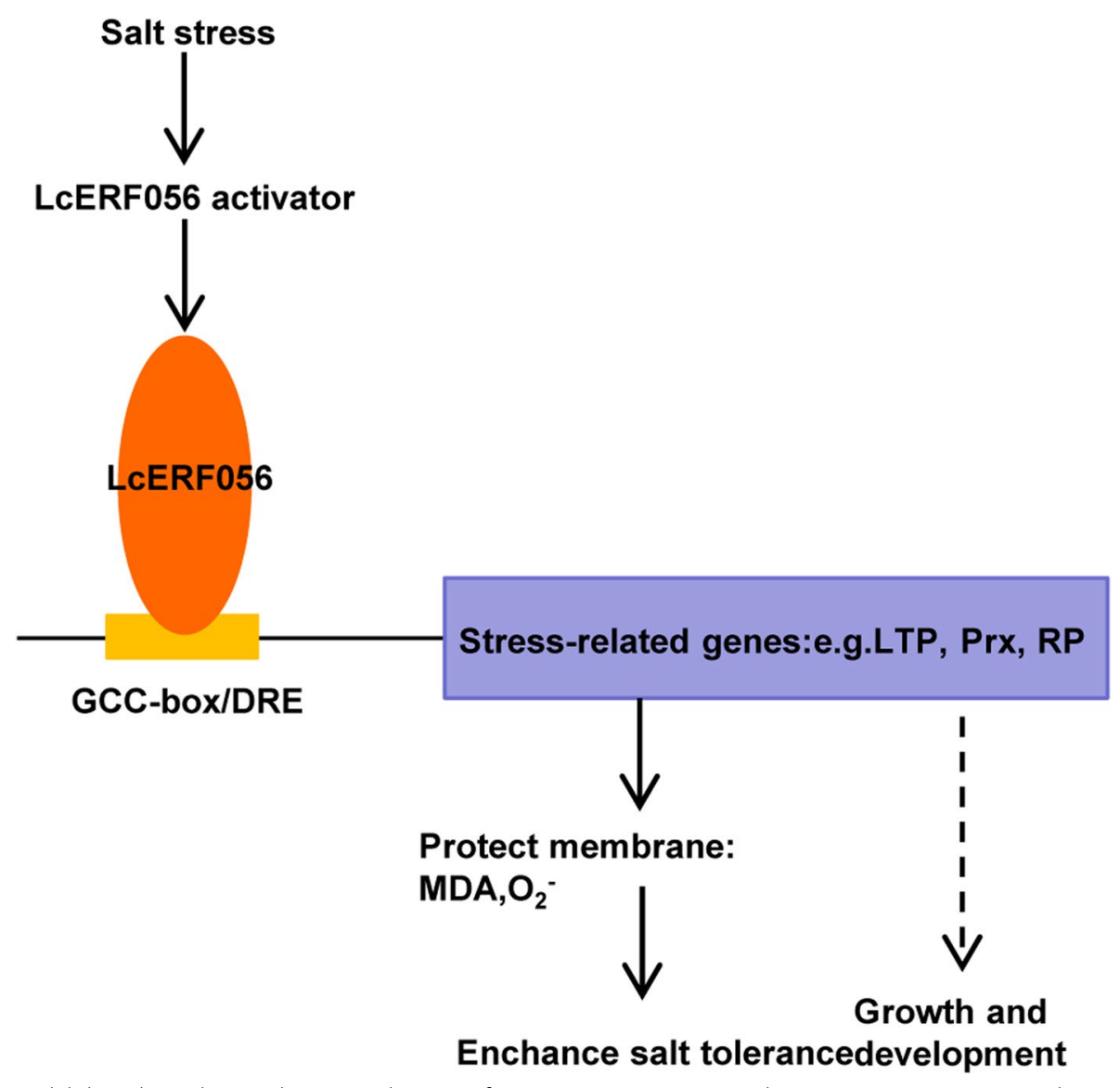

Fig. 7 A proposed model describing the regulation mechanism of LCERF056 in response to salt stress. LCERF056 activates the transcription of ROS-related genes such as LCLTP and LCPrx by directly binding to GCC-box or DRE element in the promoter and reducing reactive oxygen species, malondialdehyde (MDA), and relative electrolyte leakage. Therefore, it confers increased tolerance to salt stress in L. corniculatus 
factor LcERF056 could directly upregulate ROSrelated genes such as $L c L T P, L c P r x$, and $L c R P$ by binding to cis-element GCC box or DRE element in the promoter and reducing ROS, MDA, and relative electrolyte leakage. In this way, it conferred increased tolerance to salt stress in L. corniculatus.

\section{Conclusions}

In this study, the key salt-responsive LcERF056 belonging to B3-1 (IX) subfamily was cloned and characterised, which was considerably upregulated by salt treatment and localised in nuclei as a transcriptional activator. Furthermore, LcERF056-OE transgenic Arabidopsis and L. corniculatus lines had significantly increased tolerance to salt treatment compared with WT or RNAi transgenic lines at the phenotypic and physiological levels. LcERF056 could bind to cis-element GCC box or DRE of ROSrelated genes such as $L c L T P, L c P r x$, and $L c R P$ as demonstrated through transcriptome analysis, ChIP-qPCR, and $\mathrm{Y} 1 \mathrm{H}$ assay. These results suggested that the key regulator LCERF056 plays important roles in salt tolerance by modulating ROS-related genes. Therefore, it may be a useful target for engineering salt-stress-tolerant $L$. corniculatus or other crops.

\section{Materials and methods}

\section{Plant materials and growth conditions}

The seeds of WT L. corniculatus L. cv Leo were purchased from Beijing Clover Seed \& Turf Co. (Beijing, China), and Arabidopsis thaliana ecotype Columbia-0 were preserved in our laboratory. The plants were cultured at $25 \pm 2{ }^{\circ} \mathrm{C}$ in a growth chamber with a photoperiod of $16 \mathrm{~h} / 8 \mathrm{~h}$ (light intensity $=130-150 \mu \mathrm{E} / \mathrm{m}^{2} / \mathrm{s}$ and $50 \mu \mathrm{E} / \mathrm{m}^{2} / \mathrm{s}$, respectively).

\section{Gene cloning and sequence analysis of LCERF056}

The genomic and full cDNA sequences of LcERF056 were amplified using the primers no. 1 (Table S1), and the PCR products were sequenced using ShengGong (Shanghai, China). A multiple alignment based on the amino acid sequences between LcERF056 and other ERF family members was performed using ClustalX2 [34]. Further, the phylogenetic tree was constructed using MEGA 5.0 [35]. The conserved domains and motifs were assessed using SMART [36].

\section{RNA extraction and expression pattern analysis}

Total RNA was extracted from the seedings or different tissues of $L$. corniculatus by using an RNAprep Pure plant kit (Tiangen Biotech, Beijing, China). RTPCR or qPCR was performed according to the procedure described in previous studies [11,37]. The $L j U b i$ gene (AW720576) was used as an internal standard. At least three biological replicates were used. All primer sequences are listed in Table S6.

\section{Subcellular localisation and transactivation assay of LCERF056}

To construct the 35S::LcERF056-GFP expression plasmid, the CDS of the LCERF056 was inserted into the binary vector p163-GFP. Both 35S::GFP and the recombinant vectors were transiently expressed in Arabidopsis protoplasts, as previously described [38]. Then the protoplasts were incubated in dark for at least $16 \mathrm{~h}$. The GFP fluorescence was observed using a confocal laser scanning microscope (Zeiss LSM700).

Saccharomyces cerevisiae yeast strain AH109 and vector pDEST32 (Invitrogen, Carlsbad, CA) were used for studying the transactivation of $L C E R F 056$. The CDS of LCERF056 was cloned into pDEST32 vector, and the constructed or empty plasmids were transformed into the yeast strain AH109 as per the manufacturer's instructions (Clontech, Palo Alto, CA). The transformants were cultured on $\mathrm{SD} /-\mathrm{Leu}$ and $\mathrm{SD} /-\mathrm{Leu} /-\mathrm{His} /-$ Ade + 3-Amino-1, 2, 4-triazole (3-AT) solid medium. $\beta$-Galactosidase filter-lifted test was measured according to the Yeast Protocols Handbook (Clontech).

\section{Plasmid construction and genetic transformation}

The primers used in constructing vectors are listed in Supplemental Table S1. The full-length coding region of LCERF056 was inserted into the binary vector $\mathrm{pH} 7 \mathrm{WG} 2 \mathrm{D}$ and pK7GW1WG2 to generate 35S:LcERF056 and RNAiLcERF056 constructs, respectively. The obtained plasmid was then introduced into the Agrobacterium tumefaciens strain GV3101. Agrobacterium bearing the plasmids was transformed into Arabidopsis Col-0 plants by using the floral dip method [39]. The plasmids were introduced into L. corniculatus cotyledon by using Agrobacteriummediated genetic transformation [40]. DNA or RNA was extracted from the transformed plants by using a DNA/ RNA extraction kit (TIANGEN, Beijing, China), and PCR or qPCR was performed to identify the transgenic lines. Expression levels of LcERF056 were calculated for different transgenic lines. Primer sequences used for PCR and qPCR are listed in Supplemental Table S2.

\section{Salt tolerance assays in LCERF056 transgenic lines}

Overall, 30-day-old soil-grown LcERF056 transgenic Arabidopsis and the WT plants were irrigated with water containing $300 \mathrm{mM} \mathrm{NaCl}$ for 21 days and monitored during the next weeks. The experiment was repeated three times.

Stem cuttings of the transgenic L. corniculatus and WT were used for phenotypic, physiological, and biochemical 
analyses. When the adventitious roots were formed, the plants were transplanted to pots containing soil mixture (nutrient soil:vermiculite, $1: 1, \mathrm{v} / \mathrm{v}$ ) and grown at $22^{\circ} \mathrm{C}$ with a photoperiod of $16 \mathrm{~h} / 8 \mathrm{~h}$ (day/night) and $65 \% \mathrm{RH}$. Eight-week-old LcERF056-RNAi and LcERF056-OE transgenic lines were watered every 2 days with $250 \mathrm{mM}$ $\mathrm{NaCl}$ for 25 days. As comparison, experiment using the WT as control plant was performed under similar conditions. All experiments were repeated three times.

\section{Measurements of the physiological and biochemical traits}

The relative moisture content (\%) was defined as (fresh weight - dry weight)/fresh weight $\times 100$. Relative electrolyte leakage was calculated using the formula: (J2-J1)/ $\mathrm{J} 2$, where $\mathrm{J} 1$ is the conductivity of samples immersed in $\mathrm{ddH}_{2} \mathrm{O}$ for $2 \mathrm{~h}$, and $\mathrm{J} 2$ is the conductivity of samples boiled for $10 \mathrm{~min}$. The accumulation of $\mathrm{O}_{2}{ }^{-}$was determined using nitro blue tetrazolium staining as per a method described previously [41]. Trypan blue staining [42] was used to visualise the degree of cell damage under salt stress. To assess the water status, the relative water content was determined following the method described by Liu et al. [43]. MDA content was determined according to the method described previously [44].

\section{RNA-Seq analysis}

The penultimate three leaves were dissected from 8-week-old seedlings from WT, RNAi, and OE lines. Samples from five siblings were pooled. Three biological repeats of RNAi lines (RNAi-4, RNAi-8, and RNAi-11) and $\mathrm{OE}$ lines (OE-9, OE-12, OE-16) were performed per genotype. Total RNA was isolated from each sample, and RNA-Seq was performed at The Beijing Genomics Institute (BGI) (Beijing, China). The differentially expressed genes were from different groups. WT-VS-OE and WTVS-RNAi indicated the plants with LcERFO56 overexpression and RNAi LcERF056 compared with WT plants, respectively.

\section{Y1H assay}

$\mathrm{Y} 1 \mathrm{H}$ assays were performed using the Matchmaker Gold Yeast One-Hybrid System (Clontech). Three tandem copies of the fragment containing GCC box of the four detection promoters were used as the bait in the $\mathrm{Y} 1 \mathrm{H}$ screens (Table S7). We generated the construct of bait with GCC box of the detection promoter in front of the reporter gene AUR1-C and construct prey of LcERF056 with antibiotic resistance gene that conferred AbA $(400 \mathrm{ng} / \mathrm{mL})$ resistance to yeast.

\section{ChIP-qPCR}

ChIP experiments were performed as previously described, with minor modifications (Kaufmann and Al.
2010). Specific antibodies targeting LcERF056 were synthesised (GenScript, Shanghai, China). In brief, 8-weekold seedlings (WT, RNAi, OE) were cross-linked with $1 \%$ formaldehyde. WT plants were used as control. The chromatin DNA fragments were isolated from their nuclei to retrieve binding DNA fragments by using rabbit polyclonal antibodies with the Magna ChIPTM A kit (Millipore). The bound DNA fragments were extracted using the Gel/PCR DNA fragments extraction kit (Millipore). The levels of the bound DNAs were measured through qPCR. Primer sequences are given in Supplemental Table S7.

\section{Statistical analysis}

The data were analysed using SPSS version 17.0. Means and standard errors were calculated to compare variables. The least significant difference test at $P<0.05$ or $<0.01$ was performed to consider statistical significance.

\section{Abbreviations}

ROS: Reactive oxygen species; AP2/ERF: APETALA2/ethylene response factor: TF: Transcription factor; OE: Overexpression; RNAi: RNA interference; qPCR: Quantitative real-time Polymerase Chain Reaction; LTP: Lipid-transfer protein. WT: Wild-type; Prx: Peroxidase; RP: Ribosomal protein; GFP: Green fluorescent protein; Y1H:Yeast one-hybrid; MDA: Malondialdehyde; GO: Gene ontology; AbA: Aureobasidin A; ChIP: Chromatin immunoprecipitation; NBT: Nitro blue tetrazolium; RWC: Relative water content.

\section{Supplementary Information}

The online version contains supplementary material available at https://doi. org/10.1186/s12870-021-03336-4.

\footnotetext{
Additional file 1 Fig. S1 Sequence analysis of LCERF056 from L. cornicula tus. (A) Nucleotide and deduced amino acid sequences of the LCERF056 protein. The conserved AP2 domain was underlined. (B) The NeighbourJoining phylogenetic tree of LCERF056 and other ERF members in plants. The complete amino acid sequences were aligned with ClustalX2, and the phylogenetic tree was constructed using the MEGA 5.0 software with 1000 bootstrap replicates. The accession number of each appended protein is as follows (in parentheses): OsERF001 (Os06g40150.1), AtERF004 (AT5G11190), GmERF083 (TC431280), OsERF098 (Os02g34260.1), LcERF056 (KC777345), AtRAP2.6 (AT1G43160), PtERF (eugene3.00031319), VvERF (GSVIVP00006201001), AtERF013 (AT2G44840), AtERF073 (AT1G72360), OsERF059 (Os10g25170.1), OsERF074 (Os05g41780.1), AtERF078 (AT3G15210), OsERF083 (Os03g64260.1), AtERF101 (AT5G47220), OsERF053 (Os01g12440.1), AtERF064 (AT4G23750). (c) Comparison of the deduced LCERF056 and AtERF13 proteins. Fig. S2 Genomic PCR and semi quantitative RT-PCR in transgenic L. corniculatus. (A) LCERF056-OE vector and LCERF056-RNAi vector. (B) Transgenic LCERF056-OE lines were confirmed by checking genes LCERF056 and hygromycin using genomic PCR. The forward primer was from $35 \mathrm{~S}$ sequence of vector, and reverse primer was from LCERF056 sequence; (C) Transgenic LCERF056-RNAi lines were confirmed by checking genomic PCR. The forward primer was from intron sequence of vector, and the reverse primer was from LCERF056 sequence; (D) Semi quantitative RT-PCR of LCERF056 in OE and RNAi transgenic lines. Fig. S3 Morphological comparison of L. corniculatus wild-type (WT) vs. LCERF056-OE and LCERF056-RNAi transgenic plants. (A) qPCR analysis of LCERF056 expression in the WT and transgenic lines. (B) Phenotype analysis of height and branches of WT, LCERF056-OE, and LCERF056-RNAi lines. Bars $=5 \mathrm{~cm}$. (C) Plant height. (D) Branch number. $P<0.05$ indicated significant difference between WT and transgenic lines. Fig. S4 The GO bar charts of DEGs in RNA-Seq data. (A) The numbers of DEGs in LCERF056OE VS WT, and LCERF056-RNAi VS WT, and LCERF056-OE VS LCERF056-RNAi.
} 
(B) The GO terms of DEGs between transgenic and WT. Fig. S5 LCERF056antibody in LCERF056-RNAi, WT and LCERFO56-OE. Table S1. Primers used for constructing vector. Table S2. Primers used for detecting transgenic L. corniculatus. Table S3. List of the 18 overlapped genes. Table S4. The $\mathrm{GO}$ terms of 18 overlapped genes. Table $\mathbf{S 5}$. Information on the primers of genes used in qPCR reactions. Table S6. GCC-box in the promoters. Table S7. $4 \times$ GCC or $4 \times$ DRE in the Y1H Table S8. Primer sequences used in ChIP-qPCR

\section{Acknowledgements}

All authors are grateful to Zhe Liang and other members of Crop Molecular Biology Center in BRI for help, advice and discussion.

\section{Authors' contributions}

ZS and YW conceived and designed the research. DW and ZS conducted experiments, $X \mathrm{XH}, \mathrm{JX}, \mathrm{LH}$ and $\mathrm{YX}$ analyzed the data. DW and ZS drafted the manuscript. $\mathrm{ZS}, \mathrm{XH}$ and $\mathrm{YT}$ revised the paper. All authors read and approved the manuscript.

\section{Funding}

This work was supported by A survey on Central China grassland forage germplasm resources (2017FY100604), National Natural Science Foundation of China (32001395), Chongqing Natural Science Foundation (stc2020jcyjmsxmX0626) and the Fundamental Research Funds for Central Non-profit Scientific Institution (1610392019004). The funding bodies provided the financial support to this research, including experimental materials \& regents, primers synthesis, genes sequencing and antibody preparation.

\section{Availability of data and materials}

All LcERFs and targets sequence information is available in the Lotus japonicus Genomics Database (http://www.kazusa.or.jp/lotus/). The data generated or analyzed during current research are included in this published article and its supplemental data files and available from the corresponding author on reasonable request.

\section{Declarations}

\section{Ethics approval and consent to participate}

Not applicable.

\section{Consent for publication}

Not applicable.

\section{Competing interests}

The authors declare that they have no conflict of interest.

\section{Author details}

'Biotechnology Research Institute, Chinese Academy of Agricultural Sciences, Beijing, China. ${ }^{2}$ Key Laboratory of Southwest China Wildlife Resources Conservation (Ministry of Education), China West Normal University, Nanchong, China. ${ }^{3}$ College of Animal Science and Technology, Hunan Agricultural University, Changsha, China. ${ }^{4}$ Hubei Key Laboratory of Animal Embryo and Molecular Breeding, Institute of Animal and Veterinary Science, Hubei Academy of Agricultural Science, Wuhan, China. ${ }^{5}$ Institute of Animal and Veterinary Science, Jiangxi Academy of Agricultural Sciences, Nanchang, China. ${ }^{6}$ ChongQing Academy of Animal Sciences, Chongqing, China. ${ }^{7}$ Huanshan group, Qingdao, China.

Received: 18 June 2021 Accepted: 10 November 2021

Published online: 29 December 2021

\section{References}

1. Mansour MMF, Ali EF. Evaluation of proline functions in saline conditions. Phytochem. 2017;140:52-68.

2. Morton MJL, Awlia M, Al-Tamimi N, Saade S, Pailles Y, Negrao S, et al. Salt stress under the scalpel-dissecting the genetics of salt tolerance. Plant J. 2019;97:148-63.
3. Feng K, Hou XL, Xing GM, Liu JX, Duan AQ, Xu ZS, Li MY, Zhuang J, Xiong AS. Advances in AP2/ERF superfamily transcription factors in plant, Crit. Rev. Biotechnol. 2020;40:6, 750-7760.

4. Li Y, Zhang H, Zhang Q, Liu Q, Zhai H, Zhao N, et al. An AP2/ERF gene, IbRAP2-12, from sweetpotato is involved in salt and drought tolerance in transgenic Arabidopsis. Plant Sci. 2019;281:19-30.

5. Feng X, Feng P, Yu H, Yu X, Sun Q, Liu S, et al. GsSnRK1 interplays with transcription factor GsERF7 from wild soybean to regulate soybean stress resistance. Plant Cell Environ. 2020;28:13726.

6. Xing L, Di Z, Yang W, Liu J, Li M, Wang X, et al. Overexpression of ERF1-V from Haynaldia villosa can enhance the resistance of wheat to powdery mildew and increase the tolerance to salt and drought stresses. Front Plant Sci. 2017:8:1948.

7. Jung H, Chung PJ, Park SH, Redillas M, Kim YS, Suh JW, et al. Overexpression of OsERF48 causes regulation of OsCML16, a calmodulin-like protein gene that enhances root growth and drought tolerance. Plant Biotechnol J. 2017:15:1295-308.

8. Scarpeci TE, Frea VS, Zanor MI, Valle EM. Overexpression of AtERF019 delays plant growth and senescence, and improves drought tolerance in Arabidopsis. J Exp Bot. 2017;68:673-85.

9. Qi W, Sun F, Wang Q, Chen M, Huang Y, Feng YQ, et al. Rice ethyleneresponse AP2/ERF factor OsEATB restricts internode elongation by down-regulating a gibberellin biosynthetic gene. Plant Physiol. 2011;157:216-28.

10. Sun ZM, Zhou ML, Xiao XG, Tang YX, Wu YM. Genome-wide analysis of AP2/ERF family genes from Lotus corniculatus shows LCERF054 enhances salt tolerance. Funct Integr Genomics. 2014a;14:453-66.

11. Sun ZM, Zhou ML, Xiao XG, Tang YX, Wu YM. Overexpression of a Lotus corniculatus AP2/ERF transcription factor gene, LCERF080, enhances tolerance to salt stress in transgenicArabidopsis. Plant Biotechnol Rep. 2014b;8:315-24.

12. Sun ZM, Zhou ML, Dan W, Tang YX, Lin M, Wu YM. Overexpression of the Lotus corniculatus soloist gene LCAP2/ERF107 enhances tolerance to salt stress. Protein pept lett. 2016;23:442-9.

13. Xu Y, Zheng $X$, Song Y, Zhu L, Yu Z, Gan L, et al. NtLTP4, a lipid transfer protein that enhances salt and drought stresses tolerance in Nicotiana tabacum. Sci Rep. 2018;8:8873.

14. Liang $X$, Liu Y, Xie L, Liu X, Wei Y, Zhou X, et al. A ribosomal protein AgRPS3aE from halophilic Aspergillus glaucus confers salt tolerance in heterologous organisms. Int J Mol Sci. 2015;16:3058-70.

15. Moin M, Bakshi A, Madhav MS, Kirti PB. Expression profiling of ribosomal protein gene family in dehydration stress responses and characterization of transgenic rice plants overexpressing RPL23A for water-use efficiency and tolerance to drought and salt stresses. Front Chem. 2017;5:97.

16. Jin T, Sun Y, Zhao R, Shan Z, Gai J, Li Y. Overexpression of peroxidase gene GsPRX9 confers salt tolerance in soybean. Int J Mol Sci. 2019;20:3745.

17. Zumajo-Cardona C, Pabon-Mora N. Evolution of the APETALA2 gene lineage in seed plants. Mol Biol Evol. 2016;33:1818-32.

18. Ishikawa M, Morishita M, Higuchi Y, Ichikawa S, Ishikawa T, Nishiyama T, et al. Physcomitrella STEMIN transcription factor induces stem cell formation with epigenetic reprogramming. Nat Plants. 2019;5:681-90.

19. Nakano T, Suzuki K, Fujimura T, Shinshi H. Genome-wide analysis of the ERF gene family in Arabidopsis and rice. Plant Physiol. 2006;140:411-32.

20. Agarwal G, Garg V, Kudapa H, Doddamani D, Pazhamala LT, Khan AW, et al. Genome-wide dissection of AP2/ERF and HSP90 gene families in five legumes and expression profiles in chickpea and pigeonpea. Plant Biotechnol J. 2016;14:1563-77.

21. Zhou X, Zhang ZL, Park J, Tyler L, Yusuke J, Qiu K, et al. The ERF11 transcription factor promotes internode elongation by activating gibberellin biosynthesis and signaling. Plant Physiol. 2016;171:2760-70.

22. Bajaj D, Upadhyaya HD, Das S, Kumar V, Gowda CL, Sharma S, et al. Identification of candidate genes for dissecting complex branch number trait in chickpea. Plant Sci. 2016;245:61-70.

23. Giuntoli B, Perata P. Group VII ethylene response factors in Arabidopsis: regulation and physiological roles. Plant Physiol. 2018;176:1143-55.

24. Phukan UJ, Jeena GS, Tripathi V, Shukla RK. MaRAP2-4, a waterloggingresponsive ERF from Mentha, regulates bidirectional sugar transporter AtSWEET10 to modulate stress response in Arabidopsis. Plant Biotechnol J. 2018;16:221-33.

25. Chakravarthy S, Tuori RP, D'Ascenzo MD, Fobert PR, Despres C, Martin GB. The tomato transcription factor Pti4 regulates defense-related gene 
expression via GCC box and non-GCC box cis elements. Plant Cell. 2003;15:3033-50.

26. Zhang $H$, Yang $Y$, Zhang Z, Chen J, Wang XC, Huang R. Expression of the ethylene response factor gene TSRF1 enhances abscisic acid responses during seedling development in tobacco. Planta. 2008;228:777-87.

27. Wang X, Li Q, Cheng C, Zhang K, Lou Q, Li J, et al. Genome-wide analysis of putative lipid transfer protein LTP_2 gene family reveals CsLTP_2 genes involved in response of cucumber against root-knot nematode (Meloidogyne incognita). Genome. 2020;6(4):225-38.

28. Safi H, Saibi W, Alaoui MM, Hmyene A, Masmoudi K, Hanin M, et al. A wheat lipid transfer protein (TdLTP4) promotes tolerance to abiotic and biotic stress in Arabidopsis thaliana. Plant Physiol Biochem. 2015;89:64-75.

29. Guo $X$, Zhang L, Wang $X$, Zhang $M, X i Y$, Wang A, et al. Overexpression of Saussurea involucrata dehydrin gene SiDHN promotes cold and drought tolerance in transgenic tomato plants. PLoS One. 2019;14:e225090.

30. Zhao J, Wang S, Qin J, Sun C, Liu F. The lipid transfer protein OsLTPL159 is involved in cold tolerance at the early seedling stage in rice. Plant Biotechnol J. 2020;18:756-69.

31. Wang M, Dai W, Du J, Ming R, Dahro B, Liu JH. ERF109 of trifoliate orange (Poncirus trifoliata (L.) Raf.) contributes to cold tolerance by directly regulating expression of $\operatorname{Prx} 1$ involved in antioxidative process. Plant Biotechnol J. 2019;17:1316-32.

32. Matamoros MA, Saiz A, Penuelas M, Bustos-Sanmamed P, Mulet JM, Barja $\mathrm{MV}$, et al. Function of glutathione peroxidases in legume root nodules. J Exp Bot. 2015;66:2979-90

33. Nagaraj S, Senthilkumar M, Ramu VS, Wang K, Mysore KS. Plant ribosomal proteins, RPL12 and RPL19, play a role in nonhost disease resistance against bacterial pathogens. Front Plant Sci. 2016;6:1192.

34. Larkin MA, Blackshields G, Brown N, Chenna R, McGettigan PA, McWilliam $H$, et al. Clustal W and Clustal X version 2.0. Bioinform. 2007:23:2947-8.

35. Tamura K, Peterson D, Peterson N, Stecher G, Nei M, Kumar S. MEGA5: molecular evolutionary genetics analysis using maximum likelihood, evolutionary distance, and maximum parsimony methods. Mol Biol Evol. 2011:28:2731-9.

36. Letunic I, Doerks T, Bork P. SMART 7: recent updates to the protein domain annotation resource. Nucleic Acids Res. 2012;40:D302-5.

37. Bennett J, Hondred D, Register JC. Keeping qRT-PCR rigorous and biologically relevant. Plant Cell Rep. 2015;34:1-3.

38. Yoo SD, Cho YH, Sheen J. Arabidopsis mesophyll protoplasts: a versatile cell system for transient gene expression analysis. Nat Protoc. 2007;2:1565-72.

39. Clough SJ, Bent AF. Floral dip: a simplified method for Agrobacteriummediated transformation of Arabidopsis thaliana. Plant J. 1998;16:735-43.

40. Wang D, Luo W, Khurshid M, Gao L, Sun Z, Zhou M, et al. Co-expression of PeDREB2a and KcERF improves drought and salt tolerance in transgenic lotus corniculatus. J Plant Growth Regul. 2017:1-10.

41. Fang $L C, S u, L Y, S X M, L i X B, S M X, K S K, F S, C J F, L S H, X$ HP. Expression of Vitis amurensis NAC26 in Arabidopsis enhances drought tolerance by modulating jasmonic acid synthesis. J Exp Bot 2016;67:2829-2845.

42. Shen H, Zhong X, Zhao F, Wang Y, Yan B, Li Q, et al. Overexpression of receptor-like kinase ERECTA improves thermotolerance in rice and tomato. Nat Biotechnol. 2015;33:996-1003.

43. Liu H, Wang Y, Zhou $X$, Wang C, Wang C, Fu J, et al. Overexpression of a harpin-encoding gene popW from Ralstonia solanacearum primed antioxidant defenses with enhanced drought tolerance in tobacco plants. Plant Cell Rep. 2016;35:1333-44.

44. Wang Q, Qi W, Wang Y, Sun F, Qian X, Luo X, et al. Isolation and identification of an AP2/ERF factor that binds an allelic cis-element of rice gene LRK6. Genet Res. 2011;93:319-32.

\section{Publisher's Note}

Springer Nature remains neutral with regard to jurisdictional claims in published maps and institutional affiliations. 